\title{
Lamin heart disease with ventricular noncompaction and complex atrial septal arrhythmias: successful use of charge density mapping.
}

Christoph Scharf ${ }^{1}$, Stefano Caselli ${ }^{1}$, Lam Dang ${ }^{1}$, Kurt Mayer ${ }^{2}$, and Christine Attenhofer Jost $^{1}$

${ }^{1}$ Hirslanden Klinik im Park

${ }^{2}$ Kantonsspital Graubunden

January 24, 2022

\begin{abstract}
Lamin A/C Mutations are important to recognized for therapeutic and prognostic reasons. We present the clinical cases of two sisters with Lamin A mutation presenting with complex atrial arrhythmias, atrioventricular block and ventricular arrhythmias, associated with ventricular noncompaction and abnormal pulmonary vein anatomy. The successful treatment of complex irregular atrial arrhythmias by the use of noncontact dipole density mapping and radiofrequency ablation (RFA) is described in both sisters with long-term follow up results.
\end{abstract}

Lamin heart disease with ventricular noncompaction and complex atrial septal arrhythmias: successful use of charge density mapping.

Stefano Caselli ${ }^{1}, \mathrm{MD}, \mathrm{PhD} ; \mathrm{Lam} \mathrm{Dang}^{1}$, MSc, PhD; Kurt Mayer ${ }^{2}, \mathrm{MD}$; Christine Attenhofer Jost ${ }^{1}$, MD and Christoph Scharf ${ }^{1}$, MD

From:

1) Herzgefässmedizin, Hirslanden Klinik im Park, Zürich, Switzerland.

2) Kurt Meyer clinical practice, Chur, Switzerland

Conflict of interest statement :

Stefano Caselli, Kurt Mayer and Christine Attenhofer Jost: none to report;

Lam Dang and Christoph Scharf: founders and stockholder of Acutus Medical

Funding: none

Corresponding Author :

Christoph Scharf MD

Hezgefässmedizin, Hirslanden Klinik im Park,

Seestrasse 247; 8038 Zürich, Switzerland

christoph.scharf@gmail.com

Abstract 
Lamin A/C Mutations are important to recognized for therapeutic and prognostic reasons. We present the clinical cases of two sisters with Lamin A mutation presenting with complex atrial arrhythmias, atrioventricular block and ventricular arrhythmias, associated with ventricular noncompaction and abnormal pulmonary vein anatomy. The successful treatment of complex irregular atrial arrhythmias by the use of noncontact dipole density mapping and radiofrequency ablation (RFA) is described in both sisters with long-term follow up results.

\section{Case report patient $A$}

This female patient was diagnosed with left ventricular non-compaction, left ventricular ejection fraction (LVEF 0.52) and frequent (30\%) premature ventricular contractions (PVC) at the age of 37years. The MRI did not show any scar nor late enhancement. An electrophysiological study was performed, and the major origin site of the PVC was identified near the aortic valve and was successfully ablated. Nine years later, at the age of 46years, she presented with EF 0.47, frequent episodes of non-sustained ventricular tachycardia, together with frequent and highly symptomatic paroxysmal atrial fibrillation. The patient underwent pulmonary vein isolation and prophylactic ICD implantation. One year later a redo procedure for multiple left atrial flutters was unsuccessful, as contact mapping could not localize the irregular atrial activation patterns. An antiarrhythmic treatment with flecainide was successful, but stopped after 2 years because of decrease in

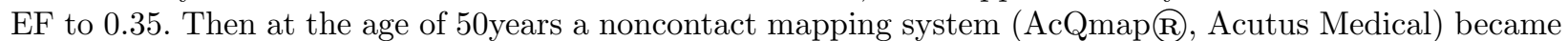
available for a redo proceure. The system has been described elsewhere (1)11. Briefly a beat to beat analysis of the electrical charge (Coulomb/area) is obtained simultaneously in the entire atrium by signal processing from 48 noncontact electrodes. The system has been validated against contact mapping in sinus rhythm and atrial fibrillation(2).

There was no activity in any pulmonary vein. A left atrial map showed a flutter with changing activation sequence originating from the interatrial septum region with a cycle length of $270 \mathrm{~ms}$. (Figure 2 upper panel left) Therefore also a right atrial map was performed, which showed the trigger at the corresponding site in the right atrial septum. During RFA at a site with very discrete electrograms (Figure 2 panel A) the flutter slowed to $365 \mathrm{msec}$ and converted to Sinus Rhythm only in the right atrium, while the left atrium continued in the arrhythmia (Figure 2 panel B). An additional left atrial map showed again the earliest triggers in the septum and further ablation there led to conversion to Sinus Rhythm (Figure 2 panel C). Afterwards a very long interatrial conduction time of $200 \mathrm{~ms}$ was observed.

During follow up of 3 years the patient remained free of atrial arrhythmias longer than 2 minutes according to the ICD interrogation, in the absence of any antiarrhythmic drug. After discontinuation of flecainide and in sinus rhythm, the EF remained stable at 0.35 . However, she experienced during follow-up repeated stroke despite anticoagulation with VKA, which were attributed to thromboembolism from the non-compacted ventricular myocardium. Therefore the anticoagulation was combined with Aspirin.

\section{Case report patient $B$}

This otherwise healthy female patient underwent RFA at the age of 39 years for atrial tachycardia from the right atrial free wall. At the time she had a normal echocardiogram with normale EF, morphology and size of both ventricles and mild biatrial enlargement and mitral regurgitation. At the age of 46 years a pacemaker was implanted for second and third degree Atrioventricular (AV) Block. One year later she had atrial flutter and underwent successful RFA. At the time prolonged atrial conduction times were noted with a transisthmus interval of $320 \mathrm{msec}$ and a split potential of $188 \mathrm{msec}$. At the age of 48 the patient presented with incessant pacemaker mediated tachycardia associated with complete anterograde AV Block; for this reason the retrograde slow pathway of the AV node was ablated.

At the age of 51years the patient had a TIA. Because of symptomatic atrial fibrillation and flutter she underwent another electrophysiological procedure. During contrast injection into the left superior pulmonary vein (LSPV) and abnormal connection to the brachiocephalic vein was found (Figure 2). This connection was subsequently closed with an Amplatzer cardiac plug. 
The pulmonary veins had no activity during an irregular atrial flutter of varying cycle length between 230 and $260 \mathrm{~ms}$ (figure $2 \mathrm{~A}$ ). Mapping with the Acutus system showed a trigger of about $0.5 \mathrm{~cm}$ diameter at the anteroseptal left atrium (Figure 2 upper panel). During Radiofrequency-Ablation conversion to sinus rhythm was observed (Figure 2B). No further atrial arrhythmia could be induced with pacing down to $250 \mathrm{~ms}$ cycle length (Figure 2C). During follow up of 1.5 years the patient remained free of relevant atrial arrhythmia (Atrial fibrillation burden $<0.7 \%$ in the pacemaker interrogation). An upgrade to an ICD was done in 2021 after pacemaker battery depletion because the Lamin Mutation hat been diagnosed with worse prognosis.

\section{Genetic Testing .}

Because of the positive family history genetic testing was performed, which revealed a heterozygtous mutation in the Exon 6 of the LMNA gene c.1129C>T(p.Arg377Cys) rs397517889 in the sisters and in the two cousins with DCM. The same mutation was found in an asymptomatic 18-year-old son of Patient A with nonsustained VT. As a consequence he is scheduled for ICD implantation.

\section{Discussion:}

This is the first case reporting the association of Lamin A/C with ventricular noncompaction and a vertical vein connection from the LSPV to the SVC. The occurrence of atrial and ventricular arrhythmias and AV block has been typically observed (3-5). Interestingly in these 2 sisters the atrial arrhythmias arose from the basal interatrial septum, closed to the area where the AV block was present. In addition significant interatrial conduction delay was present. Hence the MRI in one patient did not show any late enhancement nor scar. The disease is inherited as autosomal dominant trait with high penetrance; most importantly it may be associated with an adverse cardiac outcome including heart transplantation and malignant ventricular arrhythmia (5, 6 ). It is important for the electrophysiologist to diagnose Lamin $\mathrm{A} / \mathrm{C}$ mutations, as the prognosis is worse and therefore ICD implantation recommended in many cases(7).

Irregular atrial flutters with intraatrial conduction delay are impossible to map with contact mapping systems using summation of serial activation measurements annotated to a reference, because atrial conduction times are not constant. Instead beat to beat analysis of the simultaneous activation in the entire heart chamber shows the origin of arrhythmias at the instant without annotation. Noncontact mapping of the electrical potential has limitations set by the physics of the electrical field (1). But the development of a novel mapping system analyzing the source of the electrical field (charge density and dipole density) has allowed successful treatment of these 2 patients without major arrhythmias over a follow up of 1.5 and 3 years, as demonstrated in PM and ICD interrogation.

\section{Key Teaching Point :}

We describe several cardiac disorders associated with Lamin A mutation in the same family including, dilated cardiomyopathy, left ventricular non compaction, complex atrial and ventricular arrhythmias and a vertical vein connection.

Ablation of complex atrial arrhythmias was possible by the use of noncontact dipole density mapping and radiofrequency ablation

\section{References:}

1. Grace A, Willems S, Meyer C et al: High-resolution noncontact charge-density mapping of endocardial activation. JCI Insight. 2019 Mar 21;4(6):e126422.

2. Shi R, Parikh P, Chen Z, et al: Validation of Dipole Density Mapping During Atrial Fibrillation and Sinus Rhythm in Human Left Atrium. JACC Clin Electrophysiol. 2020 Feb;6(2):171-181

3. Captur G, Arbustini E, Bonne G, et al. Lamin and the heart, Heart 2018;104:468-479

4. Chauvel R, Derval N, Duchateau J, et al. : Persistent atrial fibrillation ablation in cardiac laminopathy: Electrophysiological findings and clinical outcomes Heart Rhythm. 2021 Mar 31:S1547-5271(21)00311-8. doi: 10.1016/j.hrthm.2021.03.040. 
5. Hasselberg NE, Haland TF, Saberniak J, Brekke PH, Berge KE, Leren TP, Edvardsen T, Haugaa KH. Lamin A/C cardiomyopathy: young onset, high penetrance, and frequent need for heart transplantation. Eur Heart J. 2018 Mar 7;39(10):853-860. doi: 10.1093/eurheartj/ehx596.

6. van Rijsingen IA, Arbustini E, Elliott PM, Mogensen J, Hermans-van Ast JF, van der Kooi AJ, van Tintelen JP, van den Berg MP, Pilotto A, Pasotti M, Jenkins S, Rowland C, Aslam U, Wilde AA, Perrot A, Pankuweit S, Zwinderman AH, Charron P, Pinto YM. Risk factors for malignant ventricular arrhythmias in lamin a/c mutation carriers a European Cohort Study . J Am Coll Cardiol 2012;59:493-500.

7. Priori SG, Blomström-Lundqvist C, Mazzanti A, Blom N, Borggrefe M, Camm J, Elliott PM, Fitzsimons D, Hatala R, Hindricks G, Kirchhof P, Kjeldsen K, Kuck KH, Hernandez-Madrid A, Nikolaou N, Norekvål TM, Spaulding C, Van Veldhuisen DJ; ESC Scientific Document Group. 2015 ESC Guidelines for the management of patients with ventricular arrhythmias and the prevention of sudden cardiac death: The Task Force for the Management of Patients with Ventricular Arrhythmias and the Prevention of Sudden Cardiac Death of the European Society of Cardiology (ESC). Endorsed by: Association for European Paediatric and Congenital Cardiology (AEPC).Eur Heart J. 2015 Nov 1;36(41):2793-2867. doi: 10.1093/eurheartj/ehv316. Epub 2015 Aug 29.

Figure 1 : echocardiographic examination of patient A at the age of 37 showing highly trabeculated and mildly dilated left ventricle. In Short axis, in end-systole a non compacted / compacted ratio of 2.4 was calculated; this in addition to a borderline ejection fraction of $52 \%$ suggested a diagnosis of left ventricular non compaction. The left ventricular diastolic function was normal. The right ventricle was not dilated and showed normal function. No valvular abnormalities were detected.

Figure 2 : electrophysiological examination with AcQmapß, Acutus Medical of Patient 1; for details see text.

Figure 3 : electrophysiological examination with AcQmapß, Acutus Medical of Patient 2; for details see text.

\section{Hosted file}

for pace.pptx available at https://authorea.com/users/457078/articles/553948-lamin-heartdisease-with-ventricular-noncompaction-and-complex-atrial-septal-arrhythmias-successfuluse-of-charge-density-mapping 